\title{
Introducing the ES Food \& Agroforestry: The Frontiers of Interdisciplinary Research that Feeds the World
}

\author{
Yue Zhang, , $^{*}$ Boce Zhang ${ }^{2, *}$ and Yangchao Luo ${ }^{3, *}$ \\ Received: 25 September 2020; Accepted: 01 October 2020. \\ Article type: Editorial article.
}

It is hard to imagine any other more important subject than food for humanity. Food, as the most basic necessity, provides nutrients and energy, and regulates vital body functions. It is not only a basic need and right, but also a great source of enjoyment. With tremendous economic developments and humanitarian interventions that uplift the standard of living in the past decades, the population percentage suffering from hunger has constantly been declining globally. Nowadays, food is arguably the safest and the most abundant, diverse, convenient, and readily accessible since the primordial time. However, according to the 2020 Fact Sheets published by the World Health Organization, about $30 \%$ of the world population is suffering from malnutrition including undernutrition, inadequate vitamins or minerals, overweight and obesity, which lead to diet-related noncommunicable diseases. ${ }^{[1]}$ In particular, the prevalence of metabolic diseases, such as cardiovascular diseases and diabetes caused by unhealthy or imbalanced dietary patterns, is rapidly growing in developed countries. Therefore, there has been a drastic change in research interests among the food industry, academia, and regulatory agencies. Collectively, the research community needs to address some of the most critical questions and challenges. For instance, how to craft and market a new genre of tasty and healthy food; how to advance food production and processing technologies to be more organic, safe, sustainable, and environmental-friendly; how to engage and educate the

${ }^{I}$ School of Food Science and Biotechnology, Zhejiang Gongshang

University, Hangzhou 310018, China

${ }^{2}$ Department of Biomedical and Nutritional Sciences, University of

Massachusetts, Lowell, MA 01854, United States

${ }^{3}$ Department of Nutritional Sciences, University of Connecticut,

Storrs, CT 06269, United States

*Email: zhangyue@zjgsu.edu.cn (Y. Zhang),boce_zhang@uml.edu (B.

Zhang),yangchao.luo@uconn.edu (Y. Luo) public to boost acceptance of new technologies, like GMO and nanotechnology; and how to innovate and support the future of humanity at the dawn of a new era of space exploration, etc.

Besides the aforementioned challenges, the food, agriculture and forestry in the twenty-first century could also face existential threats from climate change and farmland shortages. Agroforestry plays a critical role in mitigating or adapting to climate change, restoring land, protecting biodiversity, and enhancing food security. Agroforestry systems are becoming increasingly pertinent to agriculture, food, and related industries. However, there are also many challenges facing agroforestry practices, such as forest degradation and natural forest fires. It is vital for society to balance and develop synergy between food productivity and agroforestry sustainability. ${ }^{[2]}$ It is also imperative to publicize cutting-edge advances in agroforestry research and practices, while raising questions, particularly regarding the effectiveness of agroforestry to enhance food and environmental security around the world. However, we find that the connection and synergy among food, agriculture, and forestry are rarely emphasized in the established journals in either food science or agroforestry field.

ES Food \& Agroforestry is dedicated to exploring high-quality and cutting-edge original multidisciplinary research encompassing science, technology, and engineering of food, agriculture, forestry, and their interdisciplinary fields (Fig. 1), such as the relationship between sustainable agriculture and food production, integration of food security through smart agroforestry model, etc. ${ }^{[3]}$ The editorial board of the ES Food \& Agroforestry holds a strong commitment to providing an open-access platform for the scientific community to share their latest significant, novel, and high impact findings across all aspects of food, agriculture, and forestry. It is our privilege to help these groundbreaking discoveries reach the public, the shareholders, and the scientific society. 


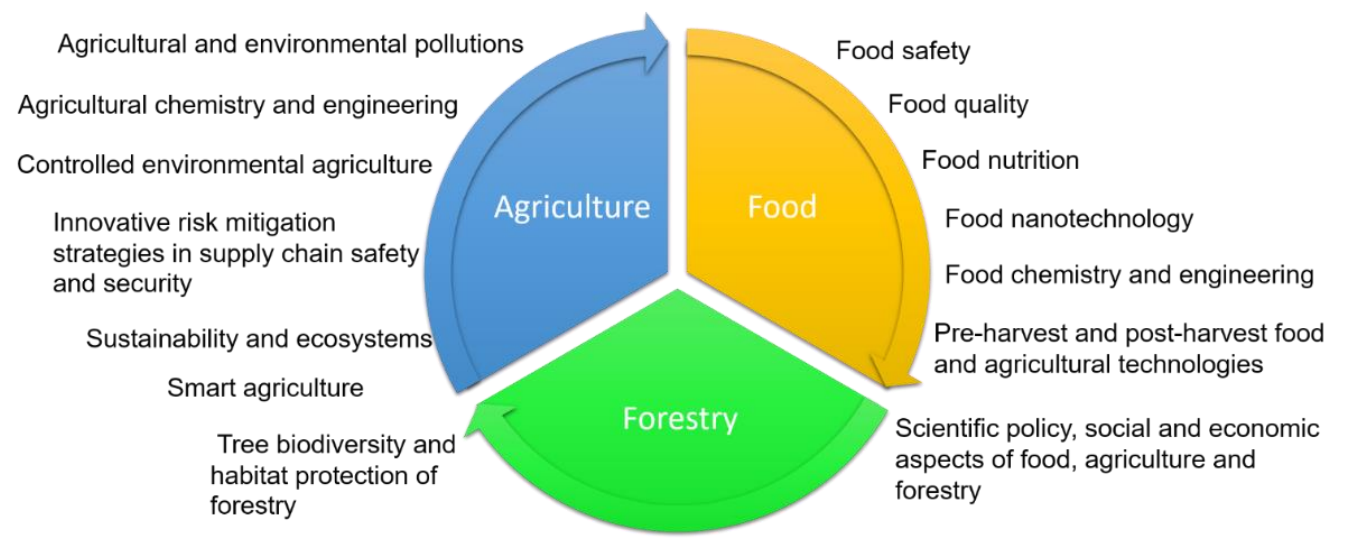

Fig. 1 The concept and the focus of ES Food \& Agroforestry.

\section{Supporting information}

Not applicable.

\section{Conflict of Interest}

There are no conflicts of interest to declare.

\section{References:}

[1] World Health Organization. 2020 Facts Sheets Malnutrition

https://www.who.int/news-room/fact-sheets/detail/malnutrition.

[2] F. Montagnini, Introduction: Challenges for Agroforestry in the New Millennium. In Integrating Landscapes: Agroforestry for Biodiversity Conservation and Food Sovereignty, Springer, 2017, 3-10.

[3] N. Sharma, B. Bohra, N. Pragya, R. Ciannella, P. Dobie, S. Lehmann, Food and Energy Security, 2016, 5, 165-183.

\section{Editor Information}

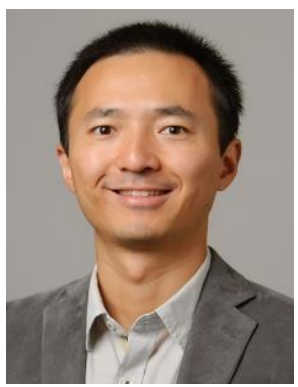

\section{Yangchao Luo}

(Editor-in-Chief)

Dr. Yangchao Luo is an Assistant Professor in the Department of Nutritional Sciences at the University of Connecticut (UConn). Dr. Luo obtained his B.S. from Hunan Agricultural University in 2006 and M.S. from China Agricultural University in 2009, followed by Ph.D. from the University of Maryland in 2012. Dr. Luo worked as a postdoctoral research associate at the University of Tennessee for one year before joining UConn in 2014. Dr. Luo's research is highly interdisciplinary and his laboratory applies materials science and engineering principles to understand physical and chemical interactions among natural biomaterials at the nanoscale and design novel nano-delivery systems for bioactive compounds. Dr. Luo is an active scholar editing multiple journals to serve the scientific community worldwide. Besides serving as the Editor-in-Chief for ES Food and Agroforestry (Engineered Science Publisher), he also edits the International Journal of Biological Macromolecules (Editor, Elsevier), the Journal of Agriculture and Food Research (Editor-in-Chief, Elsevier), and the Engineered Science (Associate Editor, Engineered
Science Publisher).

Email:yangchao.luo@uconn.edu

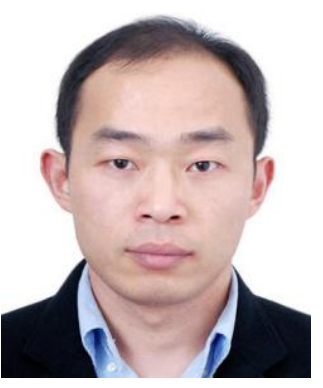

Hongbing Deng (Associate Editor)

Dr. Hongbing Deng is a Full Professor in the School of Resource and Environmental Science at Wuhan University. Dr. Deng obtained his B.S., M.S. and Ph.D. from Wuhan University from 1999 to 2010. Dr. Deng worked in the college of food science and technology at Huazhong Agricultural University from 2010 to 2012 before joining Wuhan University. Dr. Deng focuses his research on the biomass-resource chemistry and biotechnology, biomass based high value-added materials and natural biopolymer nanofibers.

Email: hbdeng@whu.edu.cn

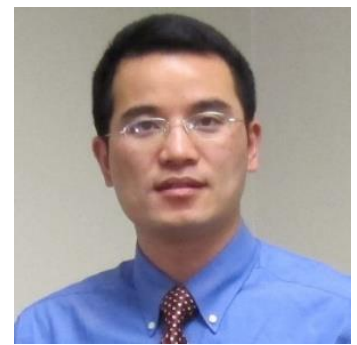

Hongshun Yang (Associate Editor)

Dr. Hongshun Yang is an Associate Professor in the Department of Food Science and Technology at the National University of Singapore (NUS). He obtained his Ph.D. in Refrigeration and Cryogenics Engineering from Shanghai Jiao Tong University in 2005 and worked as a Research Fellow at Auburn University from 2006 to 2008. He obtained his second Ph.D. in Food Science from the University of Minnesota in 2012 and was a faculty research assistant at the University of Maryland before joining NUS in 2013. Dr. Yang's research interests include food processing, safety engineering and foodomics, especially in seafood, fruit and vegetables, and cereal products. He is an Editor of LWT-Food Science and Technology, Section Editor for International Journal of Agricultural and Biological Engineering and an editorial member for Journal of Food Science. He is Chair Designate of IFT Aquatic Food Products Division.

Email: fstynghs@nus.edu.sg 


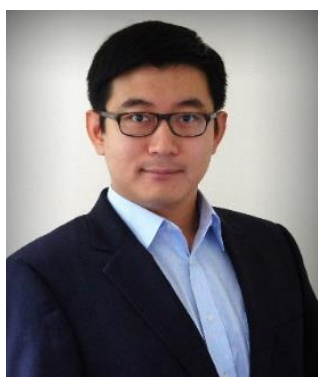

\section{Boce Zhang} (Associate Editor)

Dr. Boce Zhang is an Assistant Professor in the Department of Biomedical and Nutritional Sciences at the University of Massachusetts, Lowell. Dr. Zhang obtained his B.S. in Chemistry from the Tsinghua University in 2008 and a Ph.D. in Nutrition and Food Science from the University of Maryland, College Park in 2012. Dr. Zhang was an ORISE fellow at the U.S. Department of Agriculture prior to joining UMass Lowell. Dr. Zhang pioneers in translational innovation in food safety that encompasses microbiology, biointerface sciences, biosensor, AI-driven smart food safety, etc. He devotes his research efforts to translate advanced technologies into real-world impact in food safety and public wellbeing.

Email: boce_zhang@uml.edu

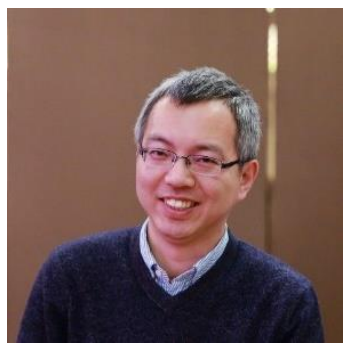

\section{Hui Zhang}

(Associate Editor)

Dr. Hui Zhang is an Associate Professor at Department of Food Science and Nutrition, Zhejiang University, China. Dr. Zhang received his Ph.D. in Food Science from Zhejiang University in 2009, and finished his postdoc work at University of Hohenheim, Germany, from October 2010 to August 2012. Dr. Zhang's research focuses on physical processing of novel food structures (e.g. emulsions, gels, fibers) for bioactive encapsulation and controlled release. Dr. Zhang is currently an Associate Editor of the Journal of the Science of Food and Agriculture (Wiley), and serves as Advisory Board member of AOCS (American Oil Chemists' Society) China Section, and as professional member of IFT (Institute of Food Technologists), ACS (American Chemical Society) and CIFST (Chinese Institute of Food Science and Technology).

Email: hubert0513@zju.edu.cn

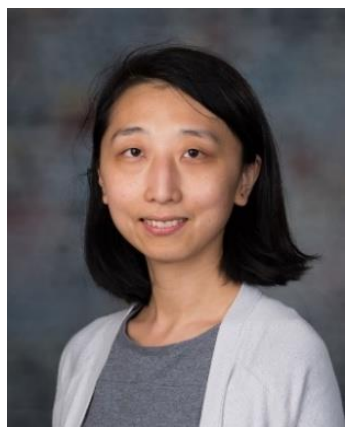

\section{Yue Zhang}

\section{(Associate Editor)}

Dr. Yue Zhang is a Professor in the School of Food Science and Biotechnology at Zhejiang Gongshang University, China. Dr. Zhang obtained her B.S in Chemistry and Ph.D. in Physical Chemistry from Wuhan University (2002-2011). She worked as a postdoc research associate at the University of Tennessee in 2011-2015, and then worked as an Assistant Professor at the University of Nebraska-Lincoln prior to joining Zhejiang Gongshang University in 2019. Dr. Zhang's research mainly focuses on the physicochemical properties of food biopolymers and the development of biopolymer-based nano-formulations for targeted delivery purpose.

Email: zhangyue@zjgsu.edu.cn

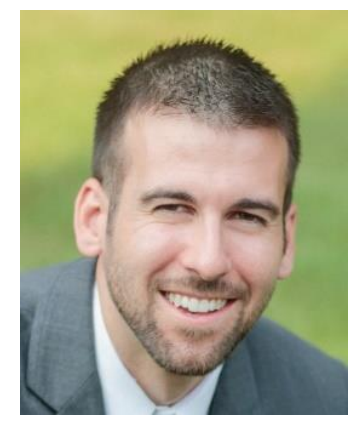

Christopher Blesso

(Executive Editor)

Dr. Christopher Blesso is an Associate Professor in the Department of Nutritional Sciences at the University of Connecticut, where he conducts research in molecular and human nutrition. His research focuses on preventing cardiometabolic diseases through dietary modification, with an emphasis in pathways related to lipid metabolism. Prior to his current position at the University of Connecticut, he obtained B.S. and M.S. degrees from Boston University in Nutritional Sciences. He then received his Ph.D. in Nutritional Sciences from the University of Connecticut, where he studied the effects of diet on lipoprotein metabolism in humans as a USDA National Needs Pre-Doctoral Fellow. He completed additional training as an American Heart Association Post-Doctoral Fellow at Wake Forest University School of Medicine, where he studied high-density lipoprotein (HDL) metabolism and atherosclerosis.

Email: christopher.blesso@uconn.edu

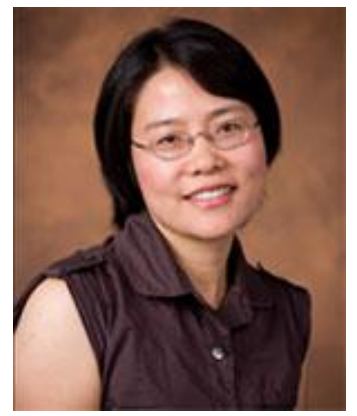

Zhaohui Tong

(Executive Editor)

Dr. Zhaohui (Julene) Tong is currently an Associate Professor in the Department of Agricultural and Biological Engineering at the University of Florida (UF). She obtained her Ph.D. degree in Chemical Engineering from Georgia Institute of Technology in 2007. She worked as a chemical engineer in Ch2mhill Co. Ltd. for two years and then joined the Department of Agricultural and Biological Engineering at UF as an assistant professor in 2010. Dr. Tong's Bioproduct Lab has conducted interdisciplinary research by applying chemistry, material science, and chemical engineering principals to produce value-added multi-functional biomaterials, biochemicals, and biofuels. Her research mainly focusses on the following aspects: 1) Synthesizing biochemicals from renewable resources (e.g. biowaste) to replace their petroleum-based counterparts; 2) Fabricating sustainable multi-functional biomaterials for environmental and human health applications; 3) Designing sustainable and energy-efficient bioprocesses to reduce environmental impacts.

Email: ztong@ufl.edu

Publisher's Note: Engineered Science Publisher remains neutral with regard to jurisdictional claims in published maps and institutional affiliations. 